\title{
The Robust Algorithm of 3D Medical Image Retrieval Based on Perceptual Hashing
}

\author{
Lian Xu \\ College of Information Science and Technology \\ Hainan University \\ Haikou, China \\ Xulian2014@hotmail.com
}

\author{
Jingbing Li* \\ College of Information Science and Technology \\ Hainan University \\ Haikou, China \\ Jingbingli2008@hotmail.com
}

\author{
Mengxing Huang \\ College of Information Science and Technology \\ Hainan University \\ Haikou, China \\ Huangmx09@163.com
}

\begin{abstract}
In order to deal with the difficulties of the heavy workloads and ambiguity of text labeling in the traditional image retrieval and the problem that the studies on 3D medical images retrieval were lacking, this paper proposes an robust algorithm based on perceptual hashing for 3D medical images retrieval. At first, the hash value of 3D medical image to be retrieved is extracted through perceptual hashing. And then the hash value database is established. Next, the NC(Normalized Cross Correlation Coefficient, NC) between the hash value of the image to be retrieved and each one in the hash value database is computed automatically. Finally the corresponding image with the highest $\mathrm{NC}$ value is retrieved and the 3D medical image retrieval is realized. The results show that this algorithm can distinguish different 3D images remarkably and has ideal robustness against Guassian noise, JPEG compression, Median filtering attacks, which is obviously better than other algorithms based on DCT, DFT. In addition, this algorithm has rapid retrieval capability and good practicability.
\end{abstract}

Key words-perceptual hashing; 3D medical images; image retrieval; hash value; $N C$

\section{INTRODUCTION}

With the rapid development of medical imaging technology and large numbers of various medical equipments emerging such as X-rays, CT(Computed Tomography), MRI(Nuclear Magnetic Resource Imaging) and so on, medical images are more and more digitized with more varieties. Nowadays hospitals produce tons of medical images data which not only reflect people's body structures, but also reveal the functions of human organs everyday. These medical images are important and objective evidences when doctors make clinical diagnosis, illness tracking, surgical planning and further studies after healing. In the process of clinical iconography diagnosis, doctors usually compare the medical images of different patients, find the images with same characteristics and make the accurate diagnosis after comprehensive analysis combined with the diagnose reports in the end.

The traditional method of medical image retrieval is based on key words, for example patients' names, admission number and the serial numbers of images etc. This method is hard to describe physical and visual characteristics of images. It consumes long time and had strong subjectivity. So this method can not meet the clinical requirements. CBMIR(Content-Based Medical Image Retrieval, CBMIR)[1] emerged in this condition. CBMIR has been applied in many organs, like breast, lung, backbone, head and so on, while these retrieval ways has some certain limitations.

Perceptual hashing is a way that a short abstract is formed through extracting and compressing perceptual contents and information from multimedia to record or mark media content, and what's more, it is further used to find the location where the media content is misrepresented. Image perceptual hashing is a big branch of perceptual hashing and it is widely used in image retrieval[2], image authentication[3], digital watermarking[4], image copy detection and so on. The feature extracting and encoding period need to contain more visual perceptual information to meet its robustness requirement. But the current research of image perceptual hashing algorithm is short of thinking about human visual properties. Most of the existing hashing algorithms [5,6] consider only the gray images. On the other hand, many hashing algorithms are sensitive to rotation. Some algorithms[7] are resilient to rotation, but their discriminative capabilities are not desire. In addition, the present research of image retrieval using perceptual hashing is mainly for $2 \mathrm{D}$ images and scarcely for $3 \mathrm{D}$ images[8].

In this paper, we proposed a robust algorithm of $3 \mathrm{D}$ medical image retrieval based on perceptual hashing. It combines image perceptual hashing and 3D DCT coefficients. This algorithm has strong robustness against Guassian noise, JPEG compression and Median filtering attacks. It shortens the time of retrieving and improves the efficiency. 


\section{FUNDAMENTAL THEORY}

\section{A. Image Perceptual Hashing Algorithm}

Perceptual hashing is a kind of one-way mapping from multimedia data set to perceptual abstract set, namely mapping the multimedia figures with same contents into a digital abstract uniquely to fulfill perceptual robustness and safety[9]. Image perceptual hashing is an important part of perceptual hashing. It can extract main information of image data and transfer them into binary sequences of hundreds or thousands of bits as fingerprints of images. According to the HVS(Human Visual System, HVS), images with different content get different hash values through perceptual hashing algorithm, while images with same content but different forms can get similar or even the same hash value. The ways to extract the perceptual unchanged feature of images are various, such as DCT coefficients of image, marginal information of image, frequency distribution histogram of image[10] and so on. The image perceptual hashing algorithm is composed of three steps as shown in the Fig. 1.

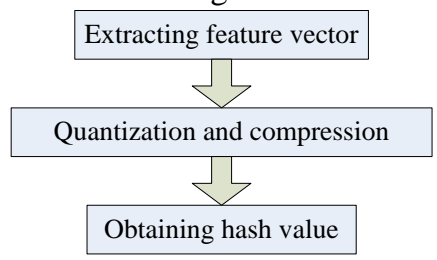

Figure 1. The process of extracting hash value of images

\section{B. Discrete Cosine Transformation}

The formula of $3 \mathrm{D}$ (three-dimensional) DCT is as follows:

$$
\begin{gathered}
F(u, \mathrm{v}, \mathrm{w})=\mathrm{c}(\mathrm{u}) \mathrm{c}(\mathrm{v}) \mathrm{c}(\mathrm{w})\left[\sum_{x=0}^{M-1} \sum_{y=0}^{N-1} \sum_{p=0}^{P-1} f(x, y, z) .\right. \\
\left.\cos \frac{(2 x+1) u \pi}{2 M} \cos \frac{(2 y+1) v \pi}{2 N} \cos \frac{(2 z+1) w \pi}{2 P}\right]
\end{gathered}
$$

$u=0,1, \mathrm{~L}, M-1 ; \mathrm{v}=0,1, \mathrm{~L}, \mathrm{~N}-1 ; \mathrm{w}=0,1, \mathrm{~L}, \mathrm{P}-1$

In the formula,

$$
\begin{aligned}
& c(u)= \begin{cases}\sqrt{1 / M} & u=0 \\
\sqrt{1 / M} & u=1,2, \mathrm{~L}, M-1\end{cases} \\
& c(v)= \begin{cases}\sqrt{1 / N} & v=0 \\
\sqrt{2 / N} & v=1,2, \mathrm{~L}, N-1\end{cases} \\
& c(w)= \begin{cases}\sqrt{1 / P} & w=0 \\
\sqrt{2 / P} & w=1,2, \mathrm{~L}, P-1\end{cases}
\end{aligned}
$$

The formula of 3D IDCT is as follows:

$$
\begin{aligned}
& F(x, y, z)=\sum_{x=0}^{M-1} \sum_{y=0}^{N-1} \sum_{p=0}^{P-1}[c(u) c(v) c(w) f(u, v, w) . \\
& \left.\cos \frac{(2 x+1) u \pi}{2 M} \cos \frac{(2 y+1) v \pi}{2 N} \cos \frac{(2 z+1) w \pi}{2 P}\right] \\
& x=0,1, \mathrm{~L}, M-1 ; \mathrm{y}=0,1, \mathrm{~L}, \mathrm{~N}-1 ; \mathrm{z}=0,1, \mathrm{~L}, \mathrm{P}-1
\end{aligned}
$$

In which, $(\mathrm{x}, \mathrm{y}, \mathrm{z})$ is the sampling value in the spatial domain and $(\mathrm{u}, \mathrm{v}, \mathrm{w})$ is the sampling value in the frequency domain.

\section{THE ALGORITHM PROCESS}

A. Acquire the hash value of the object to be retrieved.

At first, the original 3D medical image is processed using DCT, obtaining DCT coefficients matrix. Then the first $2 \times 2 \times 2$ matrix coefficients are selected to be computed using IDCT. Finally the processed image is computed using perceptual hashing algorithm to get the hash value of the object to be retrieved. In this case, we get a sequence of numbers '00000011' which is the hash value of the original 3D medical image. After observing the TAB. I, we can find that under Guassian noise, JPEG compression, and Median filtering attacks, the hash value remains unchanged and the $\mathrm{NC}$ between it attack and the original one is 1 . We also did experiments among different $3 \mathrm{D}$ images(shown as Fig. 2(a) (f)). By observing TAB. II, we can find that NC

\begin{tabular}{|c|c|c|c|c|c|c|c|c|c|c|c|c|c|}
\hline & $\begin{array}{c}\text { Image } \\
\text { manipulation }\end{array}$ & PSNR & $\mathrm{F}(1,1,1)$ & $\mathrm{F}(1,2,1)$ & $\mathrm{F}(2,1,1)$ & $\mathrm{F}(2,2,1)$ & $\mathrm{F}(1,1,2)$ & $\mathrm{F}(1,2,2)$ & $\mathrm{F}(2,1,2)$ & $\mathrm{F}(2,2,2)$ & $\begin{array}{l}\text { Mean } \\
\text { value }\end{array}$ & $\begin{array}{c}\text { Symbol } \\
\text { sequence }\end{array}$ & $\mathrm{NC}$ \\
\hline \multirow{5}{*}{ Attacks } & $\begin{array}{l}\text { Original } \\
\text { image }\end{array}$ & & -0.874 & 7.632 & 6.265 & 47.905 & -0.708 & 13.535 & 4.989 & 49.521 & 16.033 & 00000011 & 1 \\
\hline & $\begin{array}{c}\text { Guassian } \\
\text { noise } \\
(3 \%)\end{array}$ & $8.035 \mathrm{~dB}$ & 14.593 & 20.456 & 19.928 & 51.274 & 14.063 & 24.695 & 18.399 & 50.801 & 26.646 & 00000011 & 1 \\
\hline & JPEG & & & & & & & & & & & & \\
\hline & $\begin{array}{c}\text { compression } \\
(4 \%)\end{array}$ & $52.971 \mathrm{~dB}$ & -0.873 & 7.634 & 6.265 & 47.903 & -0.707 & 13.535 & 4.993 & 49.516 & 16.033 & 00000011 & 1 \\
\hline & $\begin{array}{c}\text { Median } \\
\text { filtering } \\
{[5 \times 5]}\end{array}$ & $18.073 \mathrm{~dB}$ & -0.895 & 7.217 & 5.402 & 50.191 & -0.802 & 12.139 & 4.334 & 49.711 & 15.912 & 00000011 & 1 \\
\hline
\end{tabular}
between different 3D images is very low, which can illustrate that the hash value computed by the algorithm in this paper can reflect the main visual feature of the 3D medical image. When the 3D medical image is under modest Gussian noise, JPEG compression and Median filtering attacks, this vector remains unchanged.

TABLE I. PART OF COEFFICIENTS OF IMAGES AFTER PERCEPTUAL HASHING AND VALUES AFTER DIFFERENT ATTACKS 


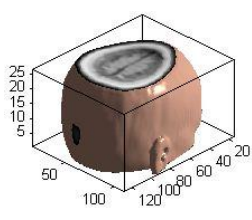

(a)

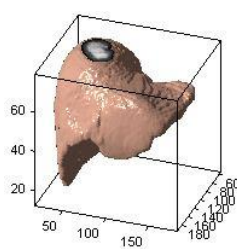

(b)

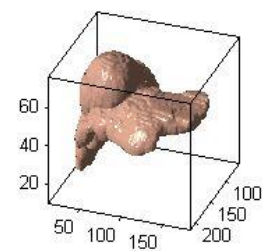

(c)

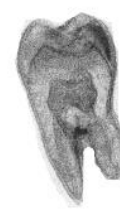

(d)

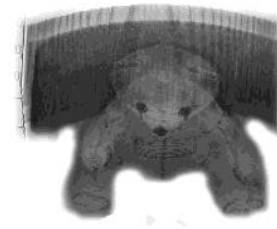

(e)

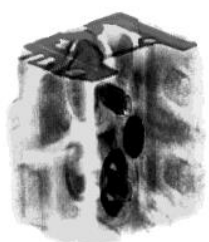

(f)

Figure 2. Different 3d medical images: (a)head; (b)liver1; (c)liver2; (d)teeth; (e)teddy bear; (f)engine

TABLE II. NC BETWEEN HASH VALUES OF DIFFERENT 3D MEDICAL IMAGES

\begin{tabular}{ccccccc}
\hline \hline & $\mathrm{Va}$ & $\mathrm{Vb}$ & $\mathrm{Vc}$ & $\mathrm{Vd}$ & $\mathrm{Ve}$ \\
\hline $\mathrm{Va}$ & 1 & 0.1079 & 0.0978 & -0.6795 & -0.0845 \\
$\mathrm{Vb}$ & 0.1079 & 1 & 0.6165 & -0.1900 & 0.04745 \\
$\mathrm{Vc}$ & 0.0978 & 0.6165 & 1 & -0.1322 & -0.0233 \\
$\mathrm{Vd}$ & -0.6795 & -0.1900 & -0.1322 & 1 & -0.0182 \\
$\mathrm{Ve}$ & -0.0845 & 0.0422 & 0.0182 & -0.0433 & 1 \\
$\mathrm{Vf}$ & -0.0845 & -0.0233 & -0.0390 & -0.0433 & 0.3623 & 0.0328 \\
\hline \hline
\end{tabular}

B. Establish hash value database of $3 D$ medical images

Step1: Obtain hash values V(j) of medical images

The 3D medical images in the image database $\mathrm{F}=\left\{\mathrm{f}_{1}, \mathrm{f}_{2}, \cdots, \mathrm{f}_{\mathrm{N}}\right\}$ are processed using $3 \mathrm{D}$ DCT separately and each DCT coefficient matrix $\mathrm{FD}(\mathrm{i}, \mathrm{j}, \mathrm{k})$ is got; the first $2 \times 2 \times 2$ coefficients are selected to be computed using IDCT and a compressed medical image $\operatorname{FID}(\mathrm{i}, \mathrm{j}, \mathrm{k})$ is got; the changed image is processed using perceptual hashing algorithm to get the hash value $\mathrm{V}(\mathrm{j})$ of the $3 \mathrm{D}$ medial image.

$$
\begin{gathered}
F D_{2}(i, j, k)=\operatorname{DCT3}(F(i, j, k)) \\
F I D(i, j, k)=\operatorname{IDCT3}\left(F D_{2}(i, j, k)\right) \\
V(j)=\operatorname{PHA}(F I D(i, j, k))
\end{gathered}
$$

Step2: Store the hash values of the 3D medical images in the hash value database $\mathrm{V}=\left\{\mathrm{V}_{1}, \mathrm{~V}_{2}, \cdots, \mathrm{V}_{\mathrm{N}}\right\}$.

\section{The automatic retrieval of $3 D$ medical images}

The model of 3D medical images retrieval based on perceptual hashing is shown in the Fig. 3.

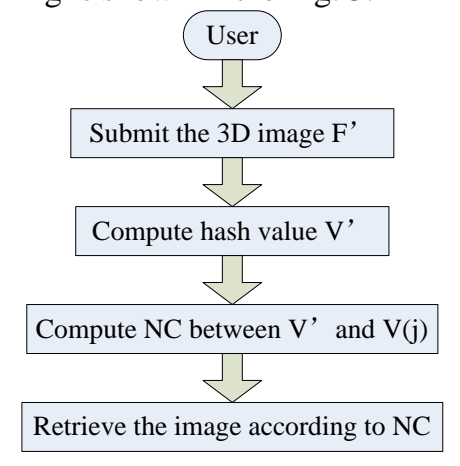

Figure 3. The 3D image retrieval model

Step1: Obtain the hash value V'(j) of the 3D medical image to be retrieved.

Provided the 3D medical image to be retrieved is $F^{\prime}(\mathrm{i}, \mathrm{j}, \mathrm{k})$ and its hash value $\mathrm{V}^{\prime}(\mathrm{j})$ is obtained using the same way of establishing hash value database in Step1.

$$
\begin{gathered}
F D_{2}^{\prime}(i, j, k)=D C T 3\left(\mathrm{~F}^{\prime}(i, j, k)\right) \\
F I D^{\prime}(i, j, k)=\operatorname{IDCT3}\left(\mathrm{FD}_{2}{ }^{\prime}(i, j, k)\right)
\end{gathered}
$$

$$
V^{\prime}(j)=\operatorname{PHA}\left(F I D^{\prime}(i, j, k)\right)
$$

Step2: Compute the NC between V(j) and V'(j).

The formula of $\mathrm{NC}$ is as follows:

$$
N C=\frac{\sum_{i} \sum_{j} W_{(i, j)} W^{\prime}{ }_{(i, j)}}{\sum_{i} \sum_{j} W^{2}{ }_{(i, j)}}
$$

$\mathrm{NC}$ is a way to evaluate the similarity between two images, the value of $\mathrm{NC}$ is larger, the similarity is higher. Each $\mathrm{NC}$ between $\mathrm{V}^{\prime}$ and $\mathrm{V}(\mathrm{j})$ in the hash value database is computed. NC with larger value represents the more similar image.

Step3: Retrieve the image according to NC.

We can retrieve the corresponding image with the highest $\mathrm{NC}$ value in the image database $\mathrm{F}$ and realize the automatic retrieval technology.

\section{THE EXPERIMENTAL RESULTS}

The simulation platform is Matlab2010a, we use 1000 groups of independent binary pseudo-random sequences (value range is +1 or 0 ), the length of every group is 512 bits. We selected a group of sequence from these 1000 groups of data (we choose the 500th group here), we put the hash value of the target image in the 500th group. We use the MRI data which belongs to Matlab to do the experiment to test and verify whether the target image can be retrieved by this algorithm when the image to be retrieved is under attacks. The original image is as shown in the Fig. 4(b), it can be expressed as $\mathrm{F}(\mathrm{i}, \mathrm{j}, \mathrm{k})$, in which $1 \leq \mathrm{i}, \mathrm{j} \leq 128,1 \leq \mathrm{k} \leq 27$, the corresponding 3D DCT coefficient matrix is $\mathrm{FD}(\mathrm{i}, \mathrm{j}, \mathrm{k})$, and the corresponding compressed image is expressed as $\mathrm{FID}(\mathrm{i}, \mathrm{j}, \mathrm{k}), \quad 1 \leq \mathrm{i}, \mathrm{j}, \mathrm{k} \leq 8$. After being processed using perceptual hashing algorithm, the hash value of the image can be obtained. Finally retrieve the similar images through computing NC. The retrieval can be realized.

In the simulation, use PSNR to evaluate the quality of images, the value of PSNR is larger, the quality of the image is better. Use $\mathrm{NC}$ to evaluate the retrieval results and set the threshold of $\mathrm{NC}$ is 0.5 , if $\mathrm{NC}$ is larger than or equal to 0.5 , the two images are similar, otherwise, they are different.

One of the slices of a 3D medical image without interference is as shown in the Fig. 4(a), it is very clear, the $3 \mathrm{D}$ medical image is as shown in the Fig. 4(b). When this 
image is the input of hash value detector, the output is as shown in the Fig. 4(c), from which we can see NC reach the

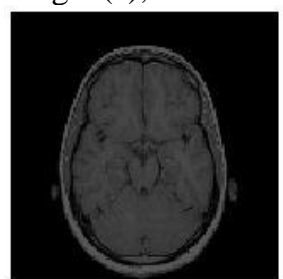

(a)

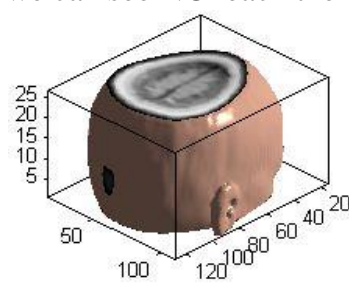

(b) peak in the 500th group, the corresponding image of the 500th hash value is as shown in the Fig. 4(d).

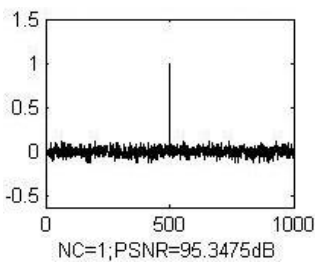

(c)

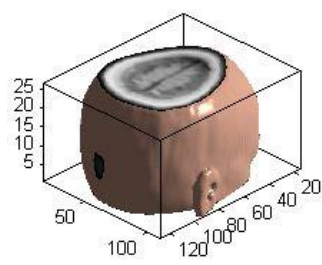

(d)

Figure 4. The original image and the retrieval result: (a)the original slice image; (b)the corresponding 3D images; (c)detector response; (d)the retrieved image.

\section{A. Interference of Gaussian Noise}

Use the function of imnoise() to add Gaussian noise to the original image. When the noise intensity is $4 \%$, the slice image is vague as shown in the Fig. 5(a), the corresponding 3D image is as shown in the Fig. 5(b). When it is the input of the hash value detector, we can see from Fig. 5(c) that the detector can retrieve the 500th hash value obviously,

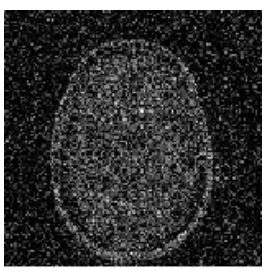

(a)

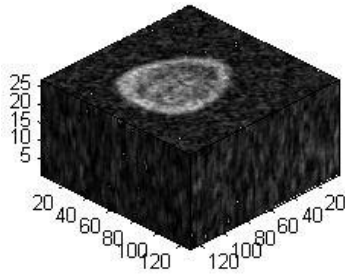

(b)

$\mathrm{NC}=0.97$. From the TAB. III, we can find when the noise intensity increases to $30 \%$ and PSNR decreases to -0.47 , $\mathrm{NC}=0.96$, the target image can also be retrieved. The retrieved 3D image is shown in the Fig. 5(d). From the TAB. III, we can find that this algorithm has strong anti-Gaussian noise capability.

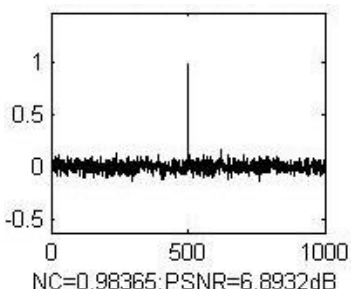

(c)

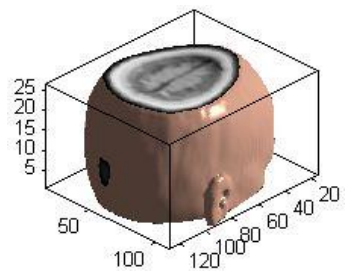

(d)

Figure 5. Under Guassian noise (the noise intensity is 4\%): (a)a slice image under Guassian noise; (b)the corresponding 3D image; (c)detector response; (d)the retrieved image.

TABLE III. THE EXPERIMENTAL DATA OF IMAGE RESISTING GUASSIAN NOISE

\begin{tabular}{cccccccc}
\hline \hline Noise intensity(\%) & 2 & 4 & 6 & 8 & 10 & 20 & 30 \\
\hline PSNR(dB) & 9.65 & 6.89 & 5.31 & 4.18 & 3.29 & 0.80 & -0.47 \\
NC & 0.98 & 0.98 & 0.97 & 0.98 & 0.95 & 0.95 & 0.96 \\
\hline \hline
\end{tabular}

\section{B. JPEG attack}

When the original image is under JPEG attack (the compression quality is 5\%), the slice image is shown as the Fig. 6(a), from which we can see clear blocking effects. $\mathrm{PSNR}=17.82 \mathrm{~dB}$. The corresponding $3 \mathrm{D}$ image is shown as

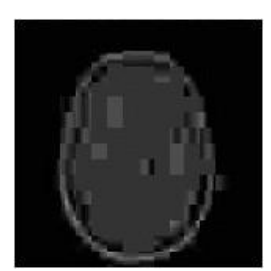

(a)

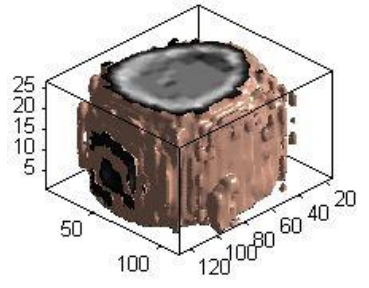

(b)

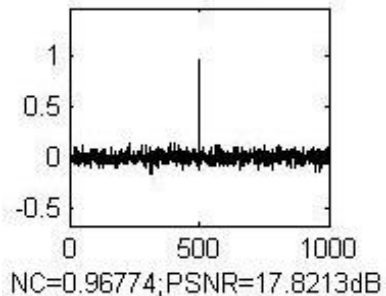

(c)

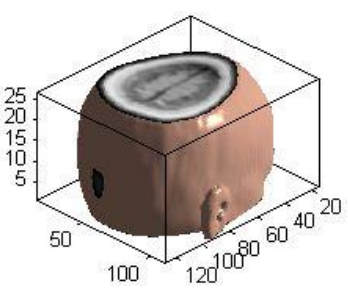

(d)

Figure 6. Under JPEG compression (the compression quality is 4\%): (a)a slice under JPEG attack; (b)the corresponding 3D image; (c)detector response; (d)the retrieved image.

TABLE IV. THE EXPERIMENTAL RESULTS OF IMAGE RESISTING JPEG COMPRESSION

\begin{tabular}{cccccccc}
\hline \hline Compression quality $(\%)$ & 1 & 4 & 10 & 30 & 50 & 70 & 90 \\
\hline PSNR(dB) & 16.56 & 17.82 & 21.20 & 24.28 & 25.88 & 27.48 & 32.99 \\
$\mathrm{NC}$ & 0.97 & 0.97 & 0.98 & 0.99 & 0.99 & 0.99 & 0.99 \\
\hline \hline
\end{tabular}




\section{Filter processing}

When the original image is under [5×5] Median filtering attack for 7 times, the slice image is vague as shown in the Fig. 7(a), the edge of the 3D image is vague too as shown in the Fig. 7(b). The most matched hash value

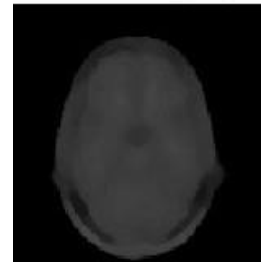

(a)

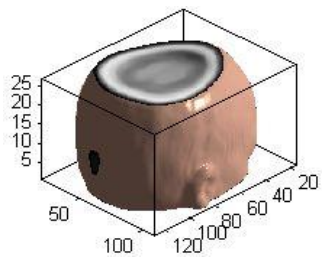

(b)

is the 500th hash value as shown in the Fig. 7(c), NC=0.98. The retrieved 3D image is shown in the Fig. 7(d). The other data of Median filtering is shown in the TAB. V, which can explain that this algorithm has strong anti-Median filtering capability.

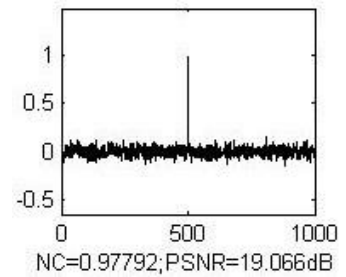

(c)

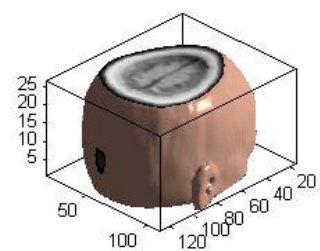

(d)

Figure 7. Under Median filtering(Median filtering[5×5] repeating 7 times): (a)a slice under Median filtering; (b)the corresponding 3D image; (c)detector response; (d)the retrieved image.

TABLE V. ThE EXPERIMENTAL DATA OF IMAGE RESISTING MEDIAN FILTERING

\begin{tabular}{|c|c|c|c|c|c|c|c|c|c|}
\hline \multirow[b]{2}{*}{ Repeating times } & \multicolumn{3}{|c|}{ Median filtering[3×3] } & \multicolumn{3}{|c|}{ Median filtering[5×5] } & \multicolumn{3}{|c|}{ Median filtering[7×7] } \\
\hline & 1 & 15 & 30 & 1 & 15 & 30 & 1 & 15 & 30 \\
\hline $\operatorname{PSNR}(\mathrm{dB})$ & 24.65 & 22.16 & 21.75 & 21.14 & 18.31 & 17.80 & 18.91 & 16.72 & 16.44 \\
\hline $\mathrm{NC}$ & 0.995 & 0.98 & 0.98 & 0.99 & 0.97 & 0.95 & 0.97 & 0.94 & 0.93 \\
\hline
\end{tabular}

\section{Algorithm Comparison}

In order to prove the capability of this algorithm in 3D image retrieval and do the quantitative analysis, we tested other algorithms (based on DCT transform domain and DFT transform domain), the results are shown in the TAB.
VI, from which we can see when an image is under same attack, perceptual hashing algorithm has better retrieval capability than the other two algorithms, it can resist normal and geometric attack effectively.

TABLE VI. COMPARISON AMONG THREE ALGORITHMS RESISTING ATTACKS

\begin{tabular}{cccc}
\hline \hline Algorithms & Based on Perceptual Hashing & Based on DCT & \multicolumn{2}{c}{ Based on DFT } \\
Attacks & NC & NC & NC \\
\hline Guassian noise(3\%) & 0.97 & 0.68 & 0.79 \\
JPEG(4\%) & 0.98 & 0.73 & 0.77 \\
Median filtering[5×5](repeating 7 times) & 0.97 & 0.88 & 0.88
\end{tabular}

\section{CONCLUSION}

This paper proposes an algorithm of 3D medical image retrieval based on perceptual hashing, which combines 3D DCT, perceptual hashing and database technologies. The experimental results show that this algorithm can distinguish different 3D images remarkably, it has ideal robustness against Gussian noise, JPEG compression, Median filtering attacks, which is better than other algorithm based on DCT, DFT. In addition, this algorithm realizes that what are stored in the database are hash values, not images, which saves the storage space widely. In addition, this method has rapid retrieval capability and good practicability.

\section{ACKNOWLEDGEMENT}

This work is supported by the National Natural Science Foundation of China (NO:61263033) and the Institutions of Higher Learning Scientific Research Special Project of Hainan (Hnkyzx2014-2) and the International Science and Technology Cooperation Project of Hainan (NO:KJHZ2014-16) and the Key Science and Technology Project of Hainan (NO:ZDXM20130078).

\section{REFERENCES}

[1] Hairong Qi, Wesley E.Snyder, Content-based image retrieval in PACS [J], Journal of Digital Image, 1999, 12(2): 81-83.
[2] SLANEY M, CASEY M. Locality-Sensitive Hashing for Finding Nearest Neighbors, IEEE Signal Processing Magazine, vol. 25, no.2, pp. 128-131, Mar. 2008.

[3] AHMED F, SIYAL M Y, ABBAS V U, A Secure and Robust Hash-Based Scheme for Image Authentication, Signal Processing, vol. 90, no.5, pp. 1457-1470, 2010.

[4] QIN C, CHANG C C, CHEN P Y. Self-Embedding Fragile Watermarking with Restoration Capability Based on Adaptive Bit Allocation Mechanism, Signal Processing, vol.92, no.4, pp. 1137-1150, 2012

[5] MONGA V, MIHCAK M K. Robust and secure image hashing via non-negative matrix factorizations. IEEE Transactions on Information Forensics and Security 2, 376-390(2007).

[6] TANG Z, Wang S, Zhang X, Wei W. Structural feature-based image hashing and similarity metric for tampering detection. Fundamental Informaticae 106, 75-91(2011).

[7] OU Y, RHEE K.H. A key-dependent secure image hashing scheme by using Radon transform. IEEE International Symposium on Intelligent Signal Processing and Communication System, pp. 595-598. IEEE Press, New York(2009)

[8] Greenspan H, Pinhas A T. Medical Image Categorization and Retrieval for PACS Using the GMM-KL Framework [J]. IEEE Transactions on Information Technology in Biomedicine, 2007, 11(2): 190-202

[9] JIAO Y H, LI M, LI Q. Key-dependent Compressed Domain Audio Hashing [J]. 8th International Conference on Intelligent Systems Design and Applications, Sept 2008 7(8): 29-32.

[10] Feng J, LI M J, Zhang H J, et al. An efficient and effective region-based image retrieval framework [J]. IEEE Transactions on Image Process, 2004, 13(5): 699-709. 